Received: 30 May 2017

Accepted: 23 October 2017

Published online: 06 November 2017

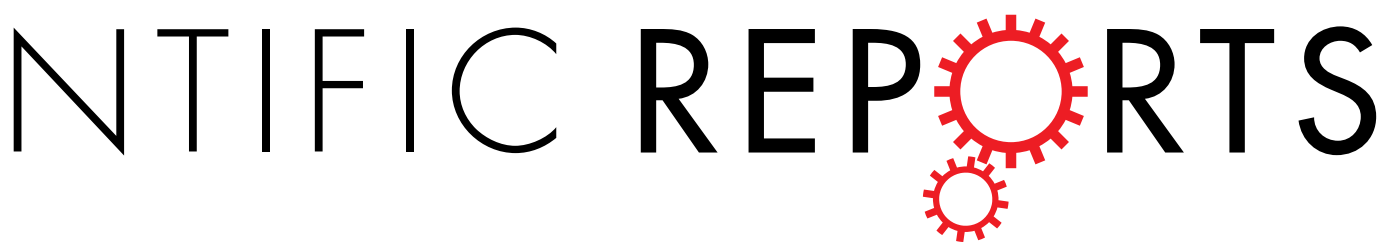

\title{
OPEN The RNA binding protein La/SS-B promotes RIG-I-mediated type I and type III IFN responses following Sendai viral infection
}

\author{
Rebecca Mahony ${ }^{1}$, Lindsay Broadbent ${ }^{2}$, Jacen S. Maier-Moore ${ }^{3}$, Ultan F. Power ${ }^{2}$ \& \\ Caroline A. Jefferies ${ }^{1,4}$
}

La/SS-B (or La) is a $48 \mathrm{kDa}$ RNA-binding protein and an autoantigen in autoimmune disorders such as systemic lupus erythematosus (SLE) and Sjögren's syndrome (SS). La involvement in regulating the type I interferon (IFN) response is controversial - acting through both positive and negative regulatory mechanisms; inhibiting the IFN response and enhancing viral growth, or directly inhibiting viral replication. We therefore sought to clarify how La regulates IFN production in response to viral infection. ShRNA knockdown of La in HEK 293T cells increased Sendai virus infection efficiency, decreased IFN- $\beta$, IFN- $\lambda 1$, and interferon-stimulated chemokine gene expression. In addition, knockdown attenuated CCL- 5 and IFN- $\lambda 1$ secretion. Thus, La has a positive role in enhancing type I and type III IFN production. Mechanistically, we show that La directly binds RIG-I and have mapped this interaction to the CARD domains of RIG-I and the N terminal domain of La. In addition, we showed that this interaction is induced following RIG-I activation and that overexpression of La enhances RIG-I-ligand binding. Together, our results demonstrate a novel role for La in mediating RIG-I-driven responses downstream of viral RNA detection, ultimately leading to enhanced type I and III IFN production and positive regulation of the anti-viral response.

Host viral detection systems rely mostly on recognition of viral nucleic acids by pattern recognition receptors (PRRs) including, RNA and DNA-sensing Toll-like receptors (TLR-3, -7, -8, -9), DNA receptors (DAI, AIM2, IFI16, DDX41) and RIG-I-like receptors (RLRs). RIG-I is an essential type I and type III IFN-inducing receptor required for the detection of negative-sense single stranded RNA viruses such Sendai virus, a member of the Paramyxoviridae family, in addition to Rhabdoviridae and Orthomyxoviridae family members ${ }^{1-3}$. Upon recognition of pathogenic RNA, an ATP-dependent conformational change is triggered in RIG-I exposing the activatory CARD domains. This allows interaction between the second CARD domain of the receptor and the CARD domain of downstream mitochondrial-associated adaptor, IPS $-1^{4-6}$. This interaction leads to assembly and activation of downstream IKK-related kinases TBK-1 and IKK- $\varepsilon$, that subsequently phosphorylate IRF-3 and IRF-7 7,8. This ultimately results in transcriptional induction of both type I and type III IFNs, which in turn leads to robust expression of IFN-stimulated genes (ISGs) ${ }^{9,10}$.

Type I IFNs, including IFN- $\alpha,-\beta,-\omega,-\kappa$ and $-\varepsilon$, act on cells via binding to the IFN- $\alpha$ receptor (IFN $\alpha R)$, comprised of an IFN $\alpha R 1$ and IFN $\alpha$ R2 heterodimer ${ }^{11,12}$. Type I IFN synthesis occurs in virtually all cell types downstream of anti-viral PRR recognition of viral RNA/DNA. Once secreted by the virally-infected cell, type I IFNs bind and activate IFN $\alpha$ R, leading to induction of interferon stimulated genes (ISGs) through activation of JAK1 and Tyk2, followed by phosphorylation of signal-transducing activators of transcription (STAT) proteins STAT1 and STAT2 ${ }^{13-16}$. ISGs, including RIG-I, TLR-3, OAS1 and OAS2, are expressed following STAT1/STAT2

\footnotetext{
${ }^{1}$ Molecular and Cellular Therapeutics, Royal College of Surgeons in Ireland, 123 St. Stephen's Green, Dublin 2, Ireland. ${ }^{2}$ Centre for Experimental Medicine, Queen's University Belfast, Medical Biology Centre, 97 Lisburn Road, Belfast, BT9 7BL, Northern Ireland. ${ }^{3}$ The University of Texas at El Paso College of Health Sciences, Clinical Laboratory Sciences Program, 500W. University Avenue, El Paso, Texas, 79968, USA. ${ }^{2}$ Division of Rheumatology, Department of Medicine and Department of Biomedical Sciences, Cedars-Sinai Medical Centre, 8700 Beverly Blvd, Los Angeles, California, 90048, USA. Correspondence and requests for materials should be addressed to C.A.J. (email: Caroline. Jefferies@cshs.org)
} 
activation, leading to the inhibition of transcription and translation of viral proteins ${ }^{17,18}$, along with induction and synthesis of MHC class I expression. This makes the cell more susceptible to CD ${ }^{+}$cytotoxic T cells ${ }^{19,20}$, activates NK cells which selectively kill virus-infected cells ${ }^{21,22}$, and leads to maturation of $\mathrm{DCs}^{23}$ and B cell responses ${ }^{20,24}$.

Functional members of the Type III IFN family, including IFN- $\lambda 1$ (IL-29), IFN- $\lambda 2$ (IL-28A) and IFN- $\lambda 3$ (IL-28B), are induced downstream of TLR-3 and RLR signalling ${ }^{25,26}$ but signal through an independent cell-surface receptor complex, consisting of IL10R2 (also called CRF2-4) and IFN- $\lambda$ R1 (also called IL-28RA) ${ }^{27,28}$. While the type I IFN receptor is ubiquitously expressed, the expression of the IFN- $\lambda \mathrm{R} 1$ component of the type III IFN receptor complex appears to be more limited and restricted to cells of epithelial origin, plasmacytoid DCs, macrophages, monocyte-derived DCs and intra-hepatic natural killer cells (NKs) ${ }^{29}$. Upon type III IFN binding to the receptor, a signal transduction cascade ensues involving activation of JAK1, JAK2 and Tyk2, followed by STATs activation and ISG expression, almost identical to that induced by type I IFN receptor ${ }^{27,30}$.

Whilst anti-viral TLRs and RLRs are well recognised for their role in inducing type I and type III IFNs, more recently RNA polymerase III (RNA pol III), an enzyme involved in the transcription of non-coding RNA, was reported to act as an anti-viral PRR by regulating type I IFN induction through generation of a RIG-I ligand ${ }^{31,32}$. RNA pol III is able to transcribe AT-rich dsDNA into the 5' ppp-dsRNA format required for recognition by RIG-I and subsequent IFN induction ${ }^{32}$. Interestingly, an autoantigen associated with systemic autoimmune disease, La/ SSB (La), binds to RNA pol III transcripts and stabilises newly-synthesised RNAs ${ }^{33-38}$. In addition to its interaction with a large variety of newly-formed RNAs, La binds a number of virus-encoded RNAs, such as adenovirus VA RNA I and VA RNA II, EBV EBER 1 \& 2 RNA, and leader RNA of negative strand RNA viruses ${ }^{39-42}$. Because La can interact with viral RNA, studies have sought to clarify its role in anti-viral immunity. Some studies proposed that $\mathrm{La}$ is manipulated by viruses in an attempt to block the anti-viral response, which it reportedly achieves by binding and sequestering the dsRNA ligand for RIG-I, thus preventing activation of the pathway ${ }^{43-45}$. On the other hand, La was also shown to promote an anti-viral response to flock house virus (FHV), although the mechanism involved was unclear ${ }^{46}$. Thus the role of La in regulating anti-viral immune responses is not well understood.

Our work described herein demonstrates a novel positive role for La in regulating type I and type III IFN responses downstream of Sendai virus infection. Our results show that knockdown of La severely impairs the ability of cells to mount an anti-viral response to Sendai virus infection, resulting in enhanced infectivity, as a result of reduced type I and III IFN production. We observed that La bound RIG-I in a ligand-inducible manner and that the CARD domains of RIG-I and RNA-binding domain of La are required for this interaction. The association between La and RIG-I promotes the interaction of RIG-I with dsRNA, thereby enhancing RIG-I-driven type I and type III IFN induction. Thus, La is required for an optimum IFN response to Sendai virus infection by binding to the anti-viral RIG-I receptor and promoting its interaction with its cognate ligand.

\section{Results}

La depletion results in enhanced Sendai virus infection efficiency and decreased Type I and Type III Interferon responses. Sendai virus $(\mathrm{SeV})$ strains are enveloped paramyxoviruses with single-stranded, non-segmented, negative sense RNA genomes. SeV strains may vary significantly in their degrees of virulence. A number of virulence factors have been mapped to either structural proteins associated with differential virus attachment and entry into host cells, or non-structural proteins implicated in immune modulation that includes antagonism of interferon signalling ${ }^{47-50}$. This study utilized a recombinant SeV expressing eGFP (rSeV/eGFP) and SeV Cantell strain, both capable of inducing type III IFN responses, while only the Cantell strain induces an additional robust type I IFN response ${ }^{51,52}$. Evidence suggests that this differential induction of type I IFN is due to the presence of defective-interfering (DI) particles in the Cantell strain ${ }^{53,54}$.

To investigate the effect of La knockdown on Sendai infectivity, HEK $293 \mathrm{~T}$ cells infected with rSeV/eGFP and transfected with either a La-specific or scrambled shRNA were evaluated by UV microscopy, using areas of comparable monolayer confluency for image analyses (Fig. 1). Supplemental Fig. 1 demonstrates successful La depletion in HEK 293 T cells, both at gene (Supplemental Fig. 1a and b) and protein (Supplemental Fig. 1c and d) levels, validating the La shRNA construct used throughout this work. Analysis of fluorescence (using Image J software) demonstrated higher eGFP coverage following rSeV/eGFP infection in cells depleted of La compared with those transfected with the scrambled control, suggesting that knockdown of La enhanced viral infectivity (Fig. 1a and b). Importantly, the increased eGFP coverage seen in La-depleted monolayers was reflected in an increase in viral titres released from these cells, compared with control cells (Fig. 1c). As La has been published to be involved in a number of cellular processes, including RNAi processing, the viability of cells transfected with scrambled or La shRNA was compared in order to ensure that La knockdown did not affect cell viability. As shown in Fig. 1d, cell viability was equivalent across the 2 different experimental conditions.

We next investigated the effect of La knockdown in HEK $293 \mathrm{~T}$ cells on type I and type III IFN induction by quantitative PCR (qPCR) following rSeV/eGFP or SeV Cantell infection. As stated above, rSeV/eGFP induced a strong type III IFN response but no type I IFN, whereas the SeV Cantell induced a robust type I IFN response in addition to type III IFNs ${ }^{51,52}$. La knockdown resulted in significant reduction of both IFN- $\beta$ and IFN- $\lambda 1$ mRNA levels following SeV Cantell infection (Fig. 2a and b), whereas a reduction in only IFN- $\lambda 1$ expression was observed in La-depleted cells following infection with $\mathrm{rSeV} / \mathrm{eGFP}$, albeit at the later time point of 48 hours post-infection (hpi) (Fig. 2e and f). Importantly, La knockdown had no effect on housekeeping gene expression as shown in Supplemental Fig. 1e. Interestingly, expression of CXCL-10 (IP-10) was significantly attenuated by La depletion following infection with both SeV strains, compared with scrambled controls (Fig. $2 \mathrm{~d}$ and h). CXCL-11 was only impaired in La-depleted cells following Cantell infection (Fig. 2c and g), suggesting that CXCL-11 induction is specifically regulated by La in the context of a type I IFN response. In contrast, the induction of CXCL-11 in response to $\mathrm{rSeV} / \mathrm{eGFP}$ was poor, with La depletion having no effect on the cells' ability to mount a response. 
La knockout

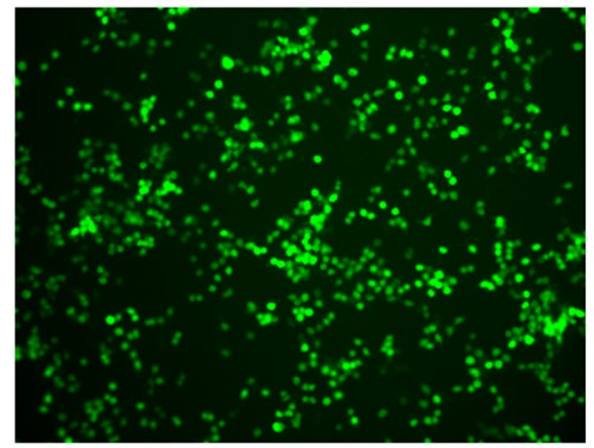

b

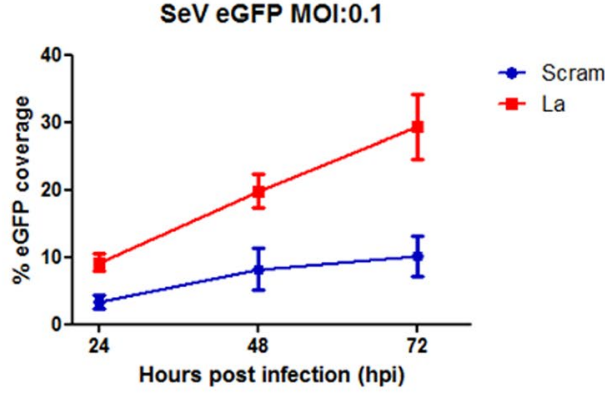

\section{Scrambled}

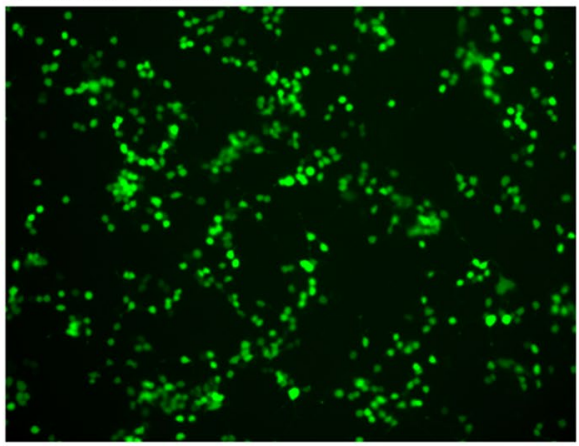

C

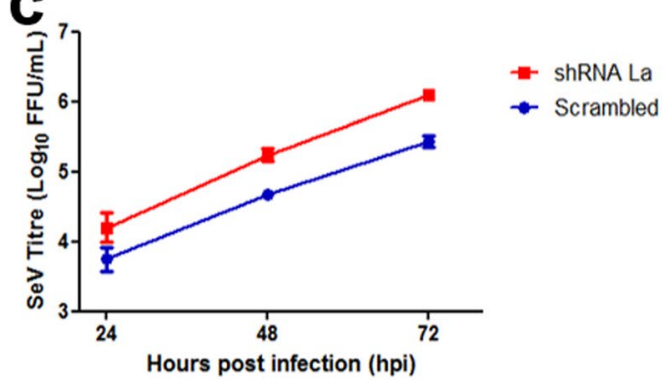

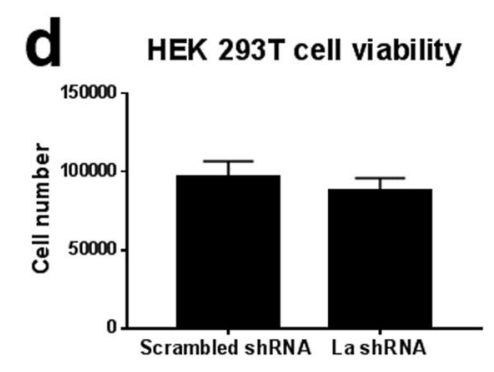

Figure 1. Depletion of La in HEK $293 \mathrm{~T}$ results in an increase in Sendai viral infection efficiency. HEK $293 \mathrm{~T}$ cells were transfected with $500 \mathrm{ng}$ of La-specific or scrambled Mission ${ }^{\circledR}$ shRNA (Sigma) for $48 \mathrm{~h}$. Cells were then infected with $\mathrm{rSeV} / \mathrm{eGFP}$ at an MOI of 0.1. (a) GFP positivity was visualised using a Nikon Eclipse TE2000-U and a Hamamatsu ORCA ER camera. (b) \% monolayer GFP-positive analysis was carried out using Image J software. (c) rSeV/eGFP titrations $(\mathrm{FFU} / \mathrm{mL}$ ) were carried out on LLC-MK2 cells. Areas under the curves were calculated and compared. Results are from two independent experiments carried out in duplicate. (d) $48 \mathrm{~h}$ post transfection, a well from each condition (Scrambled or La) was trypsinised, following which a trypan blue cell count was performed to determine the number of viable cells prior to infection. Data shown is combined average cell counts from two independent experiments.

Analysis of cytokine release following SeV infection demonstrated that CCL-5 (RANTES, a type I and III-regulated chemokine) production was impaired following SeV Cantell infection in La-depleted cells (Fig. 3a). Unsurprisingly, given that ISG expression was only induced at mRNA level following 48 hpi, no CCL-5 release was detected following infection with $\mathrm{rSeV} / \mathrm{eGFP}$ strain across the 48 hour time course of infection, nor was any significant difference observed in La-depleted cells, compared with scrambled controls (Fig. $3 c$ ). IFN- $\lambda 1$ release was completely abrogated in La-depleted cells, compared with controls, following infection with either SeV strain, further supporting our findings suggesting that $\mathrm{La}$ is crucial for the IFN response downstream of viral infection (Fig. $3 \mathrm{~b}$ and d). Assessing the effect of La knockdown on proinflammatory cytokine production in response to $\mathrm{SeV}$ Cantell infection demonstrated that La knockdown had little or no effect on the ability of the Cantell strain to induce IL-8, IL-6 or TNF- $\alpha$ (Fig. 3e-g). Importantly, our results demonstrate not only that La positively regulates type I IFN responses downstream of SeV infection, but that it also has a novel role in promoting type III I IFN induction downstream of $\mathrm{SeV}$ infection.

La enhances RIG-I binding to RNA ligand via direct interaction with the CARD-domain of RIG-I. $\mathrm{SeV}$ Cantell has been reported to rely entirely on RIG-I to elicit an anti-viral immune response ${ }^{55}$. Having demonstrated that La is required for type I and III IFN production in response to $\mathrm{SeV}$ challenge, and given its ability to bind RNA, we hypothesised that La may directly regulate RIG-I activation through regulation of RNA binding. To test this hypothesis, HEK 293 T cells were transfected with FLAG-tagged RIG-I and increasing concentrations of La from $0-2 \mu \mathrm{g}$. Cell lysates were incubated with $1 \mu \mathrm{g}$ biotin-labelled poly(I:C), and poly(I:C)-binding proteins were 
a

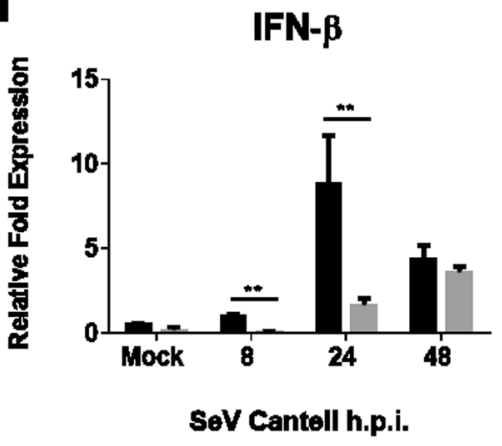

b

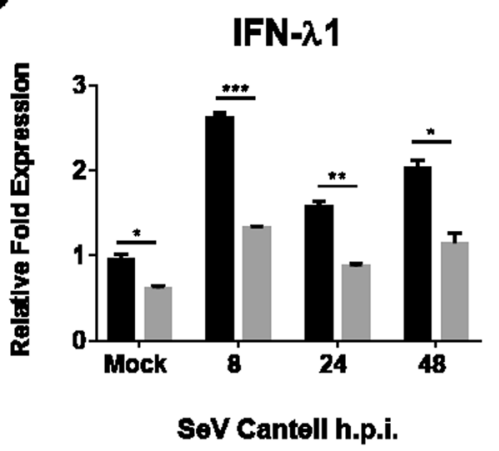

C
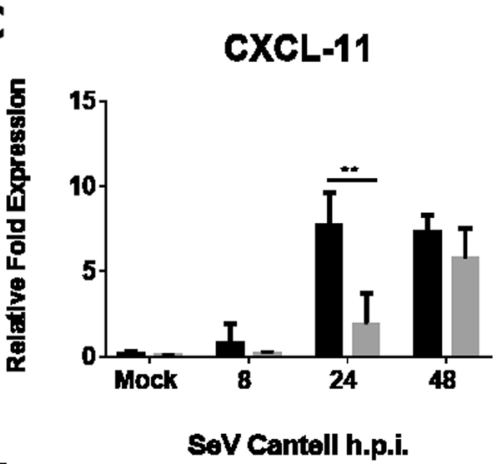

d

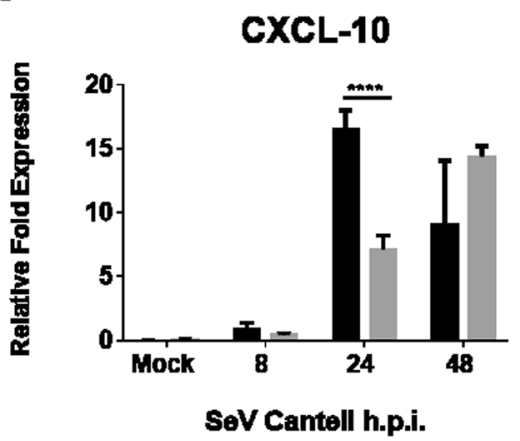

$\mathbf{e}$

IFN- $\boldsymbol{\beta}$

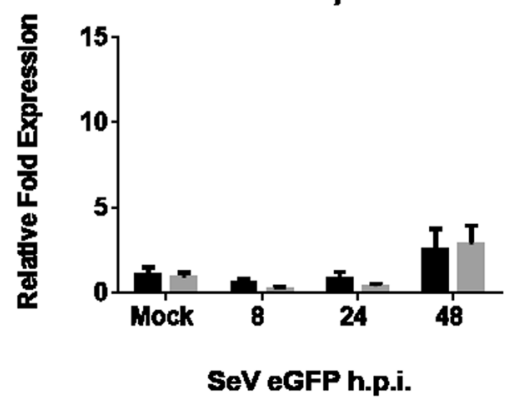

$\mathbf{f}$

IFN- $\lambda 1$

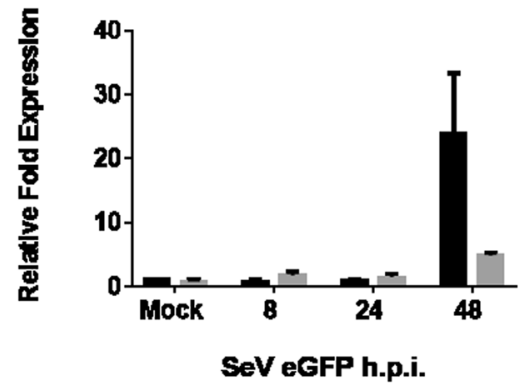

g

CXCL-11

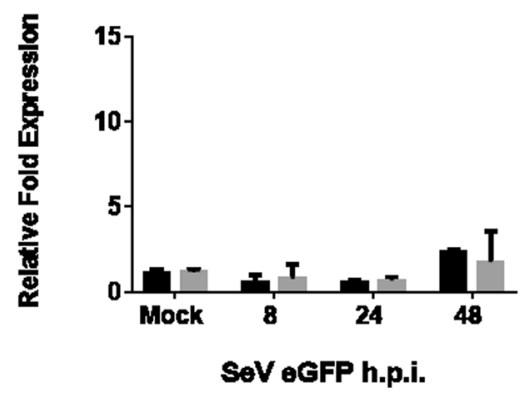

h

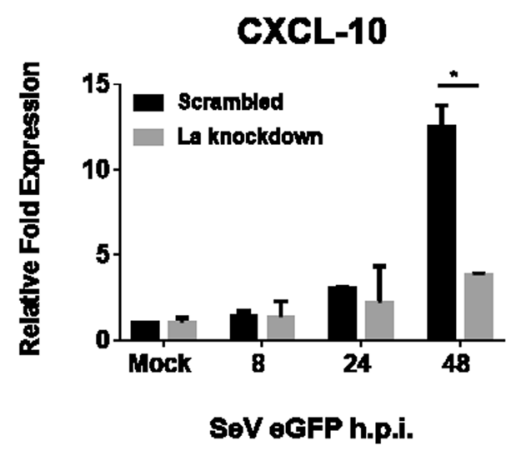

Figure 2. IFN- $\beta$, IFN- $\lambda$ and ISG mRNA expression is attenuated in La-depleted cells following SeV infection. HEK 293 T cells were transfected with 500 ng of either La-specific or scrambled Mission ${ }^{\circledR}$ shRNA (Sigma) for $48 \mathrm{~h}$ after which they were infected with SeV Cantell $(\mathbf{a}-\mathbf{d})$ or rSeV/eGFP (e-h) at an MOI of 10 and incubated for the indicated time points. IFN- $\beta(\mathbf{a}, \mathbf{e})$, IFN- $\lambda 1$ (b,f), CXCL-11 (c,g), CXCL-10 (d,h) expression was determined by RT-qPCR. Data shown are a representative of three independent experiments in each case. ${ }^{*} \mathrm{p}<0.05,{ }^{*} \mathrm{p}<0.01,{ }^{* * *} \mathrm{p}<0.001$ and ${ }^{* * *} \mathrm{p}<0.0001$, as determined by unpaired $t$-test, comparing scrambled to La shRNA at each time point. 
a

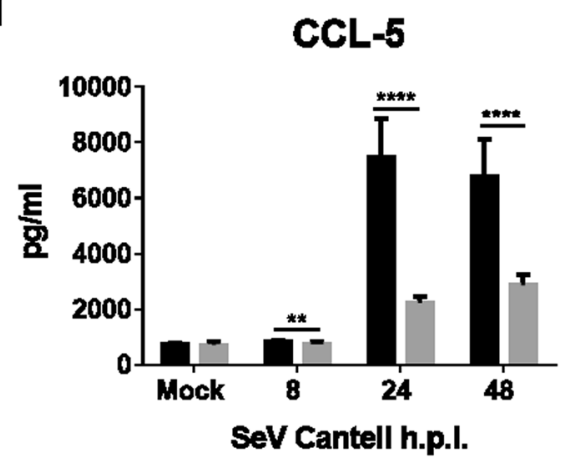

C

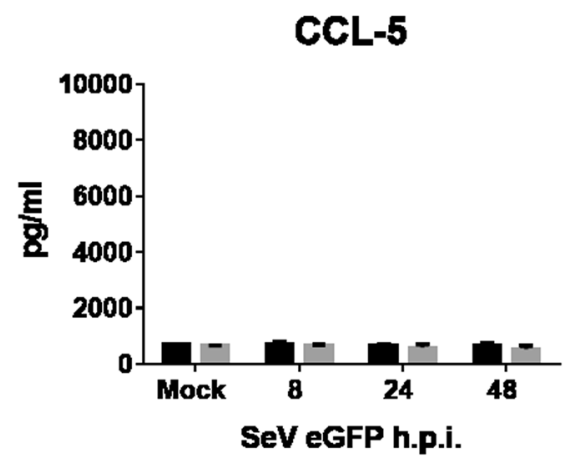

e

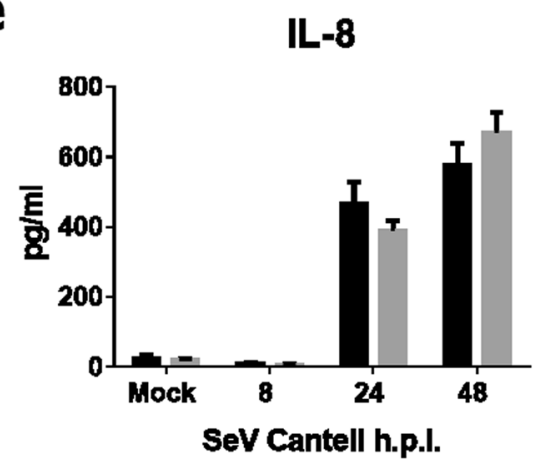

g

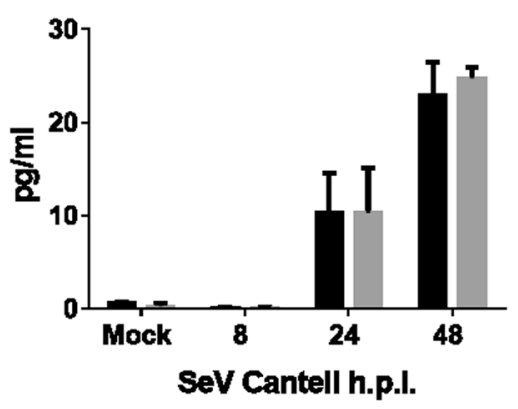

TNF- $\alpha$ b

IFN- $\lambda 1$

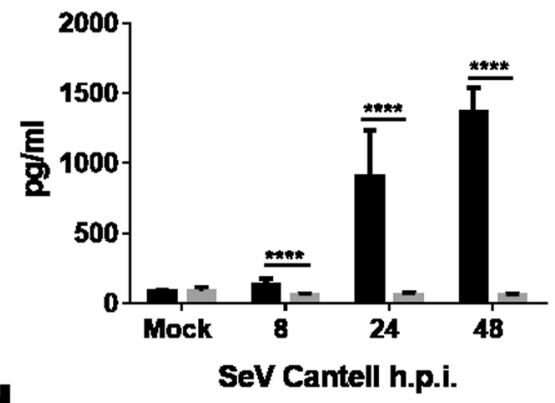

IFN- $\lambda 1$

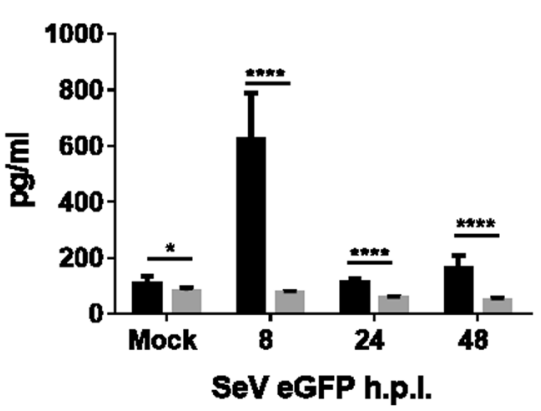

IL-6

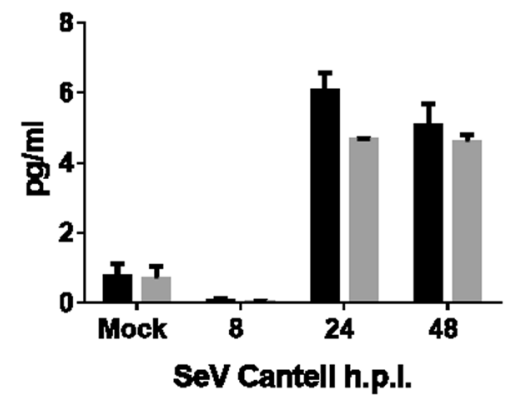


a

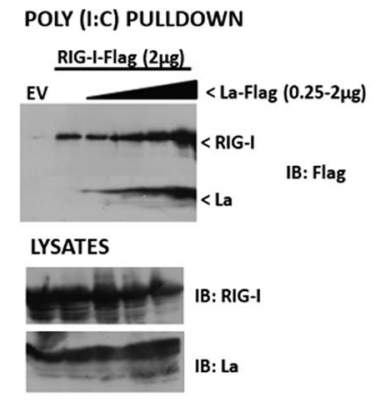

C Unstimulated 5'ppp-dsRNA 6hr

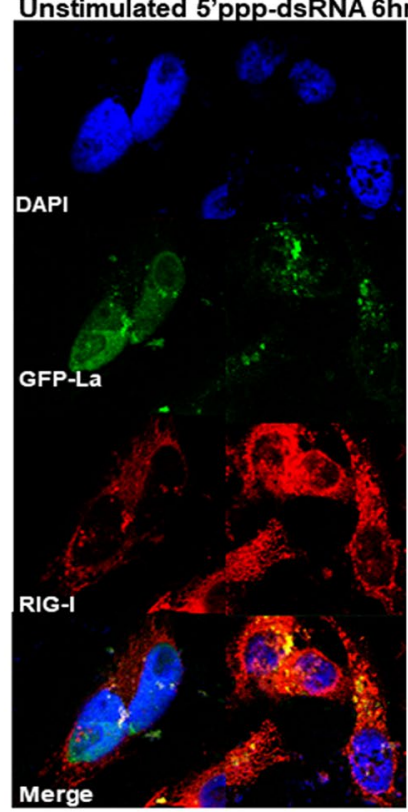

b
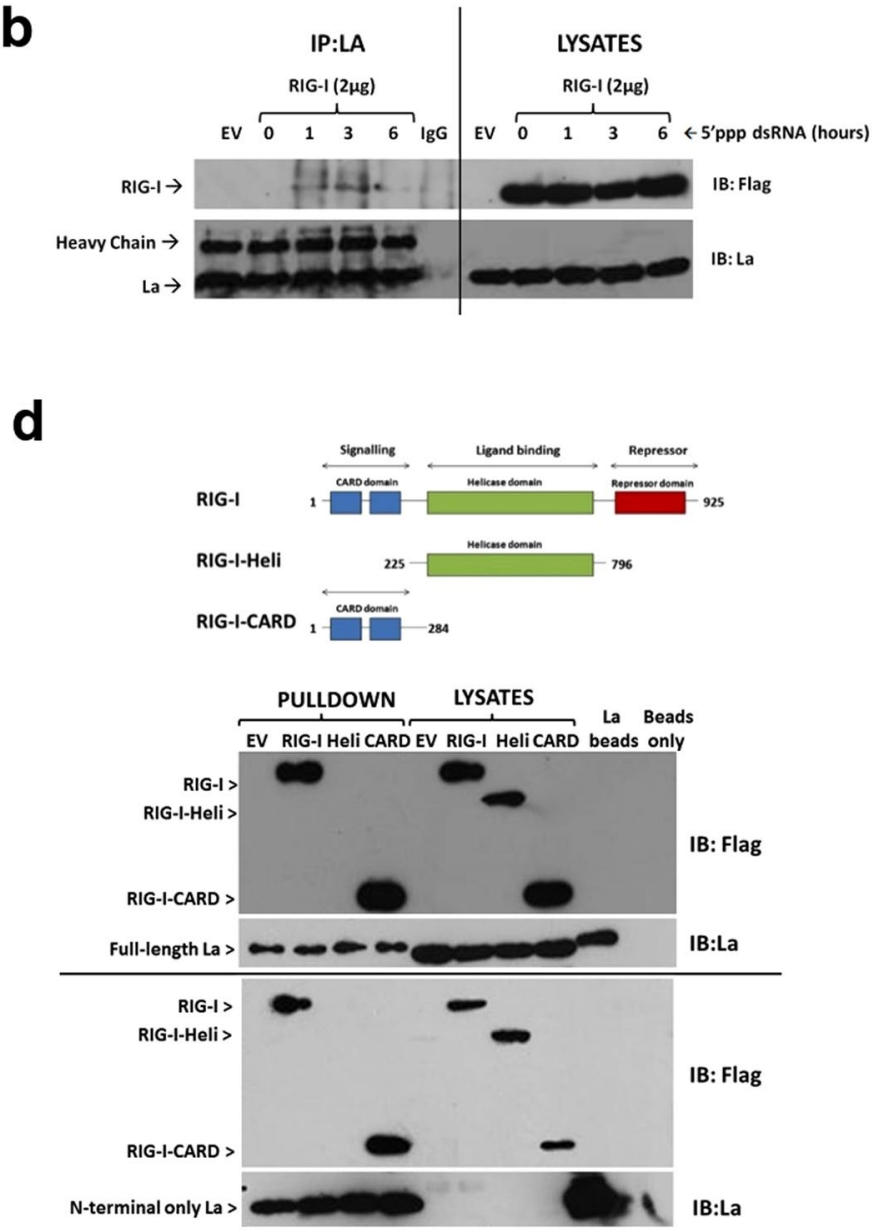

Figure 4. La enhances RIG-I binding to RNA ligand and interacts with RIG-I following 5'ppp-dsRNA stimulation. (a) HEK $293 \mathrm{~T}$ cells were transfected with $4 \mu \mathrm{g}$ EV or $2 \mu \mathrm{g}$ FLAG-tagged RIG-I with increasing FLAG-tagged La, as indicated. Analysis of the ability of FLAG-tagged La or RIG-I to bind biotinylated poly(I:C) was assessed by western blotting with anti-FLAG antibody. Expression of FLAG-tagged or RIG-I constructs in whole cell lysates was determined by western blotting with either anti-La or anti-RIG-I antibodies, as appropriate. (b) HEK $293 \mathrm{~T}$ cells were transfected as indicated and stimulated for 1, 3, or $6 \mathrm{~h}$ with $1 \mu \mathrm{g} \mathrm{5}$ pppdsRNA (Invivogen). Following immunoprecipitation of La-containing complexes with a La-specific antibody, the ability of over-expressed RIG-I to interact with endogenous La was determined by western blotting using anti-FLAG antibody. (c) HeLa cells were seeded on UV-irradiated coverslips, transfected with $2 \mu \mathrm{g}$ of GFPtagged La and $2 \mu \mathrm{g}$ FLAG-tagged RIG-I, following which they were stimulated with $1 \mu \mathrm{g} 5$ 'ppp-dsRNA for $6 \mathrm{~h}$. Immunostaining with anti-RIG-I antibody indicates that La and RIG-I co-localise following stimulation with 5'ppp-dsRNA. Both images are at 63X magnification. (d) Recombinant La was incubated with lysates prepared from HEK 293 T cells overexpressing FLAG-tagged RIG-I, FLAG-tagged RIG-I-CARD, or FLAG-tagged RIG-IHelicase (Heli), as indicated. The ability of RIG-I and La to interact was analysed by western blotting. In all cases images are representative of three independent experiments.

subsequently isolated. The ability of RIG-I to bind poly(I:C) was determined by western blotting using anti-FLAG antibody. As Fig. 4a shows, increasing concentrations of La enhanced the ability of RIG-I to bind poly(I:C). A faint lower band on the gel (corresponding to La) indicates, as would be expected from previous reports, that La was also capable of direct interaction with the RNA ligand. The lower panel of Fig. 4a demonstrates total RIG-I and La expression in the lysates; endogenous La can be observed strongly in the lane without FLAG-tagged La overexpressed, due to blotting with anti-La antibody. However, transfection with increasing concentrations of La (lanes $2-5$ ) dose-dependently increases expression, as expected. Full blots as well as statistical analysis of corresponding optical densitometry across three individual experiments are shown in Supplemental Fig. 2.

Having observed enhanced binding between RIG-I and its RNA ligand in the presence of over-expressed La, we hypothesised that La may achieve this through direct binding to RIG-I. Co-immunoprecipitation studies demonstrated an inducible interaction between La and RIG-I following stimulation of RIG-I overexpressing HEK 293 T cells with the RIG-I agonist, 5'ppp-dsRNA (Fig. 4b, Supplemental Fig. 3). This inducible interaction was statistically significant across three independent experiments (Supplemental Fig. 3d). In addition, HeLa cells over-expressing GFP-La and flag-tagged RIG-I were stimulated with 5'ppp-dsRNA, which induces 
La translocation from the nucleus to the cytoplasm where it can co-localise and interact with RIG-I (Fig. 4c). Additional confocal images demonstrating this pattern are shown in Supplemental Fig. 4 and overlap coefficients for each image are given in Supplemental Table S4.1. The translocation of La from the nucleus to the cytoplasm is consistent with data from previous studies that demonstrate similar translocation of La following viral infection $^{44,45,56}$. In addition, cells depleted of La had a reduced response to 5'ppp-dsRNA in their ability to induce IFN- $\beta$, confirming the ability of La to directly promote RIG-I induced IFN- $\beta$ expression (Supplemental Fig. 3c).

In order to determine the domains responsible for this interaction, either full-length or N-terminal-only (aa 1-204) His-tagged recombinant La was incubated with lysates from HEK $293 \mathrm{~T}$ cells over-expressing full-length RIG-I, the CARD domain-only of RIG-I, or the helicase domain-only of RIG-I. Potential interactions were analysed by western blotting. As Fig. 4d (upper panel) demonstrates, an interaction was observed between full-length La and full-length RIG-I, as expected, but also with the RIG-I deletion mutant expressing only the CARDs. No interaction was observed with helicase-only mutant of RIG-I. This indicates that the activatory CARD domains (spanning amino acids 1-284) of RIG-I are necessary and sufficient for the interaction between La and RIG-I to occur. Further analysis demonstrated that the N-terminal but not the C-terminal domain of La is required for interaction with both full length and CARD domains of RIG-I (Fig. 4d, lower panel).

Collectively, our results indicate a novel role for La as a positive regulator of type I and type III IFN production in response to $\mathrm{SeV}$ infection. We demonstrate that the mechanism of this regulation occurs through a direct interaction between La and RIG-I, which promotes RIG-I binding to its cognate ligand. Our findings not only contribute to the understanding of molecular mechanisms behind RIG-I-mediated regulation of IFN induction, but also provide valuable insight into the potential that dysregulation of La activity may contribute to over-activation of RIG-I and hence dysregulated IFN production, as observed in autoimmune diseases such as SLE.

\section{Discussion}

The induction of IFN expression is a crucially important part of the innate anti-viral immune response, not only for destruction of viral RNA and limitation of viral spread, but also for activation of adaptive immunity and selective killing of virally-infected host cells. With this work we have demonstrated a novel interaction between La and RIG-I, which results in enhanced RIG-I-RNA association. Knockdown of La resulted in increased Sendai viral infection efficiency, decreased IFN- $\beta$, IFN- $\lambda 1$ and ISG mRNA expression and attenuated CCL- 5 and IFN- $\lambda 1$ release, compared with control cells. Overall, these findings highlight an essential and novel role for La in mediating optimal type I and type III IFN responses following viral challenge in order to protect the host by both limiting viral replication and promoting the clearance of the pathogen.

Type I IFNs are the first line of defence against most types of viral infection, including the murine pathogen, $\mathrm{SeV}\left(\mathrm{DI}^{+}\right)$. They induce an anti-viral state in host cells. This is achieved by JAK/STAT pathway-mediated activation of interferon-stimulated genes (ISGs), such as RIG-I, CXCL-10, CXCL-11, OAS1 and OAS2 ${ }^{57}$. Our findings demonstrated a significant decrease in the induction of IFN- $\beta$, CXCL-10, and CXCL-11 following SeV infection upon depletion of La. While type III IFNs are structurally and genetically distinctive from type I IFNs and act through a separate receptor system, they have similar mechanisms of induction, signal transduction and biological function ${ }^{26,58}$. SeV is a potent inducer of type III IFN responses ${ }^{27,59}$. Our study identifies La as a novel positive regulator of IFN- $\lambda 1$ induction downstream of $\mathrm{SeV}$ infection. Collectively our results indicate an important role for La in inhibiting $\mathrm{SeV}$ replication by promoting both type I (as seen following Cantell infection) and type III (as seen following Cantell and rSeV/eGFP) IFN responses.

RIG-I is central to the regulation of both type I and type III IFN production as it is responsible for detection of $\mathrm{SeV}$ infection within cells. Regarding regulation of RIG-I activity, a number of proteins have been identified to play a role through post-translational modifications. For example, TRIM25 and Riplet/RNF135/REUL induce K63-linked ubiquitination within the CARD domains of RIG-I following viral infection, a modification which is necessary for interaction with IPS-1 ${ }^{60-62}$. In addition, CK2-mediated phosphorylation of RIG-I at Thr 770 and Ser 854 inhibits the anti-viral response to both hepatitis $\mathrm{C}$ virus and $\mathrm{SeV}$ and renders RIG-I inactive ${ }^{63}$. This prevents TRIM25-mediated ubiquitination of RIG-I, thereby negatively regulating the IFN response ${ }^{60}$. With this work, we have identified a novel function for the autoantigen La in enhancement of anti-viral responses. Specifically, it binds directly to the RIG-I receptor in an inducible manner and strengthens RIG-I binding to its RNA ligand, making it unique in its mechanism of action from other known RIG-I regulators, such as TRIM25 and CK2. Thus La positively regulates type I and type III IFN responses by augmenting stable RNA-RIG-I complex formation, which results in robust pathway activation. As RIG-I can also drive inflammatory gene expression through interaction with a IPS-1-CARD9-Bcl-10 complex and activation of $\mathrm{NF} \kappa \mathrm{B}^{64}$, it would appear that the interaction between La and RIG-I is able to enhance the IFN- $\beta$ response (presumably via enhancing interaction of IPS- 1 with

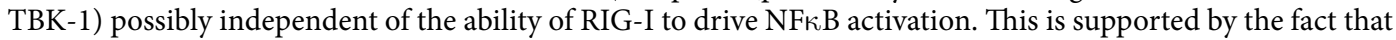
La knockdown has no effect on inflammatory gene expression downstream of RIG-I interaction, whereas IFN- $\beta$ expression is severely reduced.

Regarding a potential role for La in regulating assembly of RNA-binding complexes, Liu and colleagues ${ }^{46}$ demonstrated a role for La in RNAi processing. They reported that La associated with Ago 2 of the RISC complex in an RNA-dependent manner, thereby promoting RISC complex catalysis and RNAi processing. This finding is consistent with our data which show that La augments RIG-I binding to poly (I.:C). Importantly, deletion of the RNA-binding domain of La blocks interaction with RIG-I, underlining the RNA-binding role of La in driving RIG-I activity. In keeping with our findings that $\mathrm{La}$ is a positive regulator of viral-induced type I IFN, Liu et al. showed that La could promote the anti-viral response to flock house virus (FHV) in Drosophilia S2 cells. Indeed, La depletion resulted in increased FHV infectivity, which supports our findings that La promotes anti-viral responses to Sendai virus in HEK $293 \mathrm{~T}$ cells. In contrast, Bitko and colleagues demonstrated enhanced IFN- $\beta$ mRNA levels and decreased viral titres upon siRNA depletion of $\mathrm{La}^{43}$. In addition, Domitrovich et al. argued a role for La as a negative regulator of IFN production in the context of HCV replication, based on a 
63-67\% reduction in RNA replication in the absence of La in Huh7 cells and increased IFN- $\beta$ mRNA 10 hours post-RSV infection in the absence of $\mathrm{La}^{44}$. However, similar to our findings, both of these studies also observed an overall decrease in IFN- $\beta$ production 24 hours post-infection in La-depleted cells, suggesting that La may play a dual role in regulating anti-viral responses. Indeed, La may play a role in maintaining homeostasis in cells with respect to IFN- $\beta$ production, as evidenced by the enhanced IFN- $\beta$ expression in unstimulated cells depleted of La (Supplementary Figure 3c), whereas when RIG-I is activated, depletion of La results in substantial reduction in IFN- $\beta$ production. This indicates that the loss of La may somehow disrupt homeostatic mechanisms to maintain appropriate IFN- $\beta$ levels or indeed may inhibit viral-specific evasion mechanisms. For example, the RSV-derived NS2 protein binds to RIG-I and blocks its interaction with IPS-1, thereby preventing IRF-3 activation ${ }^{65,66}$. Thus the loss of La may disrupt the negative function of NS2 on this system, thereby contributing to the enhanced IFN- $\beta$ observed in similar studies. Extensive studies would be required to address these questions, which are outside the scope of this manuscript.

With this work, we identify a role for La in regulating IFN responses by promoting the RIG-I-mediated anti-viral response through direct association with RIG-I and enhancing RIG-I binding to its viral agonist. Importantly, our study is the first to assess the role for La in regulating type III IFN responses, with all previous studies focusing on type I IFN only. SeV infection experiments support these findings, with depletion of La resulting in increased viral infectivity and decreased type I and type III IFN responses, compared with controls. These findings highlight an important and novel role for La in the promotion of optimal type I and type III IFN responses following $\mathrm{SeV}$ challenge, serving to protect the host through limiting viral replication.

\section{Methods}

Materials. The SW5 monoclonal La antibody was generated by Professor Michael Bachmann at the Technical University of Dresden and was a kind gift from Dr. JS Maier-Moore ${ }^{67}$. All flag-tagged RIG-I plasmid constructs were a kind gift from Dr. Kate Fitzgerald (UMASS Med School, Worcester, MA). The GFP-tagged La construct was a gift from Dr. Karl Albert Brokstad (University of Bergen, Germany). Monoclonal M2 Flag antibody was purchased from Santa Cruz, pcDNA3.1 empty vector control from Invitrogen and biotin-labelled poly(I:C) from Cayla-Invivogen. A Mission ${ }^{\circledR}$ shRNA construct specific to human La, as well as a scrambled control, were purchased from Sigma.

Cell Culture. HEK $293 \mathrm{~T}$ and HeLa cell lines were cultured in Dulbecco's Modified Essential Medium (DMEM) containing stable $2 \mathrm{mM}$ L-glutamine, $10 \%$ (v/v) foetal calf serum (FCS), 100 units/ml Penicillin, $100 \mu \mathrm{g} /$ $\mathrm{ml}$ Streptomycin and $100 \mu \mathrm{g} / \mathrm{ml}$ gentamicin. LLC-MK2 cells (ECACC 85062804) were grown in minimum Eagle's medium (MEM) containing $40 \mathrm{~g} / \mathrm{ml}$ non-essential amino acids and supplemented with $10 \%$ heat inactivated FCS, $2 \mathrm{mM}$ L-glutamine, $2 \mathrm{mg} / \mathrm{ml}$ sodium carbonate, $100 \mathrm{~g} / \mathrm{ml}$ gentamicin and $1.25 \mathrm{mg} / \mathrm{ml}$ Fungizone. Cells were maintained at $37^{\circ} \mathrm{C}$ in a humidified atmosphere of $5 \% \mathrm{CO}_{2}$.

La Knockdown. HEK $293 \mathrm{~T}$ cells were seeded at $5 \times 10^{4}$ cells per $\mathrm{ml}$ and transfected the following day with $500 \mathrm{ng}$ of scrambled or La-specific shRNA (Sigma). Following $48 \mathrm{hr}$, cells were washed with PBS prior to viral infection as detailed below.

Viral Infection. The rescue and characterisation of recombinant Sendai virus expressing eGFP (rSeV/eGFP) was previously described ${ }^{68}$. The $\mathrm{SeV}$ Cantell strain, a wild type strain containing defective interfering particles, comes originally from Charles River Laboratories. Media was discarded and replaced with fresh pre-warmed DMEM supplemented with antibiotic only (no FCS), in order to limit cell growth. Cells were then infected with either rSeV/eGFP or SeV Cantell at a multiplicity of infection (MOI) of 0.1 or 10, as indicated in figure legends. One hour post-infection, inocula were removed by discarding media and replaced with DMEM supplemented with antibiotic and 1\% FCS to ensure cell survival while maintaining limited growth. At indicated time points, media was carefully removed and retained for subsequent cytokine analysis, cells were gently re-suspended in ice-cold PBS and centrifugation was carried out at $400 \times g$ for $5 \mathrm{~min}$ to pellet cells for subsequent analysis. Cells were then re-suspended in Trizol reagent for gene expression analysis or SDS sample buffer supplemented for protein expression analysis.

SeV titrations. For SeV/eGFP, a 1:10 dilution series of the sample was added to LLC-MK2 cells in MEM 1\% FBS. At 24 hpi fluorescent foci were counted. The titer is calculated as fluorescent forming units (FFU) by multiplying the average number of foci by the dilution factor at a given dilution. The dilution at which the foci were counted is equal to the inverse of the exponent of the final FFU. The titer of SeV Cantell stock was determined by plaque assay as previously described ${ }^{69}$.

Real-time polymerase chain reaction (qPCR). RNA was extracted from cell cultures using Trizol ${ }^{\mathrm{TM}}$ (Sigma) and reverse transcribed to complementary DNA using the GoScript Reverse Transcription kit (Promega), as per manufacturer's instructions. Real-time quantitative PCR investigating gene expression was performed using primers listed in Table 1, with SYBR Green Taq ReadyMix (Sigma) according to manufacturer's recommendations. Data were analyzed using an ABI Prism 7900 system (Applied Biosystems) and were normalized to $18 \mathrm{~s}$ RNA. Real-time PCR data were analyzed using the $2^{-\Delta \Delta c t} \operatorname{method}^{70}$.

Western blotting. To prepare whole cell lysates, cells were lysed in SDS buffer $(250 \mathrm{mM}$ Tris- $\mathrm{HCl}, \mathrm{pH} 6.8$, $10 \%$ SDS, $0.5 \%$ Bromophenol blue, $50 \%$ Glycerol, $50 \mathrm{nM} \mathrm{DTT}$ ) and boiled at $95^{\circ} \mathrm{C}$ for $10 \mathrm{~min}$. Equal quantities of whole cell lysates were resolved by electrophoresis on a denaturing SDS-polyacrylamide gel according to the method of Laemmli ${ }^{71}$ and transferred to a nitrocellulose membrane. Following immunoblotting, the membrane was developed using enhanced chemiluminescent horse radish peroxidase (HRP) substrate (Millipore). 


\begin{tabular}{|l|l|l|}
\hline Primer Name & Primer Sequence & Product Size(bp) \\
\hline La sense & GAAGGAGAGGTGGAAAAAG & 372 \\
\hline La anti-sense & AAGCCCCGCAAACAAAAG & \\
\hline IFN $-\beta$ sense & CTAGCACTGGCTGGAATGAGA & 217 \\
\hline IFN $-\beta$ anti-sense & CTGACTATGGTCCAGGCACA & \\
\hline $18 \mathrm{~S}$ sense & TTGACGGAAGGGCACCACCA & 131 \\
\hline $18 \mathrm{~S}$ anti-sense & GCACCACCACCCACGGAATCG & \\
\hline IFN- $\lambda 1$ sense & GGACGCCTTGGAAGAGTCACT & 84 \\
\hline IFN- $\lambda 1$ anti-sense & AGAAGCCTCAGGTCCCAATTC & \\
\hline CXCL- 10 sense & GGAAGCACTGCATCGATTTTG & 519 \\
\hline CXCL- 10 anti-sense & CAGAATCGAAGGCCATCAAGA & \\
\hline CXCL- 11 sense & GCCTTGGCTGTGATATTGTGTG & 686 \\
\hline CXCL- 11 anti-sense & CACTTTCACTGCTTTTACCCCAG & \\
\hline
\end{tabular}

Table 1. Human primers used in this study.

Co-immunoprecipitation. Cells were treated as described in the figure legends, lysed in EBC lysis buffer (Deionised water containing $50 \mathrm{mM}$ Tris ( $\mathrm{pH} 8.0$ ), $150 \mathrm{mM} \mathrm{NaCl}, 1 \%$ Nonidet $\mathrm{P} 40,0.5 \%(\mathrm{w} / \mathrm{v}$ ) sodium deoxycholate and $0.1 \%$ SDS containing $1 \mathrm{mM}$ Sodium orthovanadate $\left(\mathrm{Na}_{3} \mathrm{VO}_{4}\right), 1 \mathrm{mM}$ Phenylmethylsulfonylfluoride (PMSF), $1 \mathrm{mM}$ Potassium fluoride (KF), ) and incubated with SW5 anti-La antibody coupled to protein A sepharose beads. Thereafter, immune complexes were washed and re-suspended in SDS sample buffer for western blot analysis.

Recombinant protein pull-downs. Following a gentle wash with ice-cold PBS, lysates were prepared by addition of EBC buffer. After sonication and centrifugation, the supernatant was incubated with $50 \mu l$ Nickel agarose beads coupled to approximately $1 \mu \mathrm{g}$ either full length $(8 \mathrm{~A})$ or $\mathrm{N}$-terminal truncated $(7 \mathrm{~A})$ recombinant $\mathrm{La}$ (Dr. J. Maier-Moore), for $2 \mathrm{~h}$ on rotation at $4{ }^{\circ} \mathrm{C}$. After incubation, nickel agarose was washed three times with EBC buffer by gentle inversion and centrifugation at 5,000 $\times \mathrm{g}$. Beads were then re-suspended in SDS sample buffer for western blot analysis.

Enzyme-linked Immunosorbance Assay (ELISA). $\quad$ ELISAs were carried out using DuoSet ${ }^{\circledR}$ ELISA Development Kit for human CCL-5 (Rantes) or human IFN- $\lambda 1$ (IL-29) (eBioscience) as per the manufacturer's instructions.

RNA Immunoprecipitation. Cells were seeded and transfected as indicated in figure legends. Cells were lysed for $20 \mathrm{~min}$ at $4{ }^{\circ} \mathrm{C}$ on rotation in freshly prepared sterile RNA Immunoprecipitation (RIP) buffer ( $150 \mathrm{mM}$ $\mathrm{KCl}, 25 \mathrm{mM}$ Tris pH 7.4, $5 \mathrm{mM}$ EDTA, $0.5 \mathrm{mM}$ DTT, 0.5\% NP-40) supplemented with protease inhibitors and SUPERase RNAse inhibitor (Sigma). Samples were sonicated for $15 \mathrm{sec}$, cell debris was pelleted by centrifugation and cell supernatants were transferred to fresh tubes. One $\mu \mathrm{g}$ of biotin-labelled poly(I:C) (Invivogen) was added, followed by incubation at $4^{\circ} \mathrm{C}$ for $1-2 \mathrm{~h}$. The samples were then added to $50 \mu \mathrm{l}$ of pre-washed UltraLink NeutrAvidin beads (Pierce) and incubated for $1 \mathrm{~h}$ at $4^{\circ} \mathrm{C}$. The resulting immune complexes were then washed with RIPA buffer and re-suspended in $30 \mu \mathrm{l}$ SDS sample buffer, prior to western blot analysis.

Immunofluorescence. HeLa cells were seeded at $1 \times 10^{5} /$ well on coverslips, transfected and stimulated as indicated in figure legends. Cells were fixed and permeabilised with $4 \%$ paraformaldehyde and $0.2 \%(\mathrm{v} / \mathrm{v})$ Triton X-100 in PBS. After washing, cells were blocked in PBS with $1.2 \%$ (w/v) Fish Gelatin and $100 \mathrm{mM}$ glycine and then incubated at $37^{\circ} \mathrm{C}$ for $1 \mathrm{~h}$ with the primary antibody of interest at 1:100 dilution in blocking buffer, followed by detection with the appropriate fluorescently labelled secondary antibody at 1:200 dilution. Cells were mounted and nuclei stained using ProLong ${ }^{\circledR}$ Gold anti-fade reagent with DAPI. Cells were imaged using the LSM 710 System (Carl Zeiss) and analysed for co-localisation using Zen 9 software.

Statistical analysis. All data was analysed using GraphPad Prism (version 7) statistical software package, as specified. Statistical comparison between groups was carried out using tests described in figure legends. Data is graphically represented as mean $+/-$ standard error of the mean (SEM). $P$ values less than or equal to 0.05 were considered significant.

\section{Data Availability.}

- No datasets were generated or analysed during the current study.

- All data generated or analysed during this study are included in this published article (and its Supplementary files).

\section{References}

1. Yoneyama, M. et al. Shared and unique functions of the DExD/H-box helicases RIG-I, MDA5, and LGP2 in antiviral innate immunity. Immunol 175, 2851-2858 (2005).

2. Kato, H. et al. Cell type-specific involvement of RIG-I in antiviral response. Immunity 23, 19-28 (2005).

3. Loo, Y. M. et al. Distinct RIG-I and MDA5 signaling by RNA viruses in innate immunity. J Virol 82, 335-345 (2008).

4. Kawai, T. et al. IPS-1, an adaptor triggering RIG-I- and Mda5-mediated type I interferon induction. Nature immunology 6, 981-988 (2005). 
5. Meylan, E. et al. Cardif is an adaptor protein in the RIG-I antiviral pathway and is targeted by hepatitis C virus. Nature 437, 1167-1172 (2005).

6. Kumar, H. et al. Essential role of IPS-1 in innate immune responses against RNA viruses. J Exp Med 203, 1795-1803 (2006).

7. Sharma, S. et al. Triggering the interferon antiviral response through an IKK-related pathway. Science 300, 1148-1151 (2003).

8. Fitzgerald, K. A. et al. IKKepsilon and TBK1 are essential components of the IRF3 signaling pathway. Nature immunology 4, 491-496 (2003).

9. Lin, R., Mamane, Y. \& Hiscott, J. Structural and Functional Analysis of Interferon Regulatory Factor 3: Localization of the Transactivation and Autoinhibitory Domains. Molecular and Cellular Biology 19, 2465-2474 (1999).

10. Kumar, K. P., McBride, K. M., Weaver, B. K., Dingwall, C. \& Reich, N. C. Regulated Nuclear-Cytoplasmic Localization of Interferon Regulatory Factor 3, a Subunit of Double-Stranded RNA-Activated Factor 1. Molecular and Cellular Biology 20, 4159-4168 (2000).

11. Isaacs, A. \& Lindenmann, J. Virus interference. I. The interferon. Proc R Soc Lond B Biol Sci 147, 258-267 (1957).

12. Isaacs, A., Lindenmann, J. \& Valentine, R. C. Virus interference. II. Some properties of interferon. Proc R Soc Lond B Biol Sci 147, 268-273 (1957).

13. O'Shea, J. J., Notarangelo, L. D., Johnston, J. A. \& Candotti, F. Advances in the understanding of cytokine signal transduction: the role of Jaks and STATs in immunoregulation and the pathogenesis of immunodeficiency. J Clin Immunol 17, 431-447 (1997).

14. Horvath, C. M., Stark, G. R., Kerr, I. M. \& Darnell, J. E. Jr. Interactions between STAT and non-STAT proteins in the interferonstimulated gene factor 3 transcription complex. Mol Cell Biol 16, 6957-6964 (1996)

15. Sato, M. et al. Distinct and essential roles of transcription factors IRF-3 and IRF-7 in response to viruses for IFN-alpha/beta gene induction. Immunity 13, 539-548 (2000).

16. Honda, K. et al. IRF-7 is the master regulator of type-I interferon-dependent immune responses. Nature 434, $772-777$ (2005).

17. Clemens, M. J. \& Elia, A. The double-stranded RNA-dependent protein kinase PKR: structure and function. J Interferon Cytokine Res 17, 503-524 (1997).

18. Stark, G. R., Kerr, I. M., Williams, B. R. G., Silverman, R. H. \& Schreiber, R. D. How cells respond to interferons. Annual Review of Biochemistry 67, 227-264 (1998).

19. Le Bon, A. et al. Direct Stimulation of T Cells by Type I IFN Enhances the CD8+ T Cell Response during Cross-Priming. The Journal of Immunology 176, 4682-4689 (2006).

20. Le Bon, A. et al. Cutting Edge: Enhancement of Antibody Responses Through Direct Stimulation of B and T Cells by Type I IFN. The Journal of Immunology 176, 2074-2078 (2006).

21. Trinchieri, G., Santoli, D., Granato, D. \& Perussia, B. Antagonistic effects of interferons on the cytotoxicity mediated by natural killer cells. Fed Proc 40, 2705-2710 (1981).

22. Salazar-Mather, T. P., Ishikawa, R. \& Biron, C. A. NK cell trafficking and cytokine expression in splenic compartments after IFN induction and viral infection. J Immunol 157, 3054-3064 (1996).

23. Le Bon, A. et al. Cross-priming of CD8+ T cells stimulated by virus-induced type I interferon. Nature immunology 4, 1009-1015 (2003).

24. Le Bon, A. et al. Type i interferons potently enhance humoral immunity and can promote isotype switching by stimulating dendritic cells in vivo. Immunity 14, 461-470 (2001).

25. Zhou, L. et al. Activation of toll-like receptor-3 induces interferon-lambda expression in human neuronal cells. Neuroscience 159, 629-637 (2009).

26. Onoguchi, K. et al. Viral infections activate types I and III interferon genes through a common mechanism. J Biol Chem 282, $7576-7581$ (2007).

27. Kotenko, S. V. et al. IFN-lambdas mediate antiviral protection through a distinct class II cytokine receptor complex. Nature immunology 4, 69-77 (2003).

28. Sheppard, P. et al. IL-28, IL-29 and their class II cytokine receptor IL-28R. Nature immunology 4, 63-68 (2003).

29. Sommereyns, C., Paul, S., Staeheli, P. \& Michiels, T. IFN-lambda (IFN-lambda) is expressed in a tissue-dependent fashion and primarily acts on epithelial cells in vivo. PLoS Pathog 4, e1000017 (2008).

30. Dumoutier, L., Lejeune, D., Hor, S., Fickenscher, H. \& Renauld, J. C. Cloning of a new type II cytokine receptor activating signal transducer and activator of transcription (STAT) 1, STAT2 and STAT3. Biochem J 370, 391-396 (2003).

31. Chiu, Y. H., Macmillan, J. B. \& Chen, Z. J. RNA polymerase III detects cytosolic DNA and induces type I interferons through the RIG-I pathway. Cell 138, 576-591 (2009).

32. Ablasser, A. et al. RIG-I-dependent sensing of poly(dA:dT) through the induction of an RNA polymerase III-transcribed RNA intermediate. Nature immunology 10, 1065-1072 (2009).

33. Maraia, R. J. Transcription termination factor La is also an initiation factor for RNA polymerase III. Proc Natl Acad Sci USA 93, 3383-3387 (1996)

34. Gottlieb, E. \& Steitz, J. A. The RNA binding protein La influences both the accuracy and the efficiency of RNA polymerase III transcription in vitro. EMBO J 8, 841-850 (1989).

35. Gottlieb, E. \& Steitz, J. A. Function of the mammalian La protein: evidence for its action in transcription termination by RNA polymerase III. EMBO J 8, 851-861 (1989).

36. Stefano, J. E. Purified lupus antigen La recognizes an oligouridylate stretch common to the $3^{\prime}$ termini of RNA polymerase III transcripts. Cell 36, 145-154 (1984).

37. Maraia, R. J. \& Intine, R. V. A. Recognition of Nascent RNA by the Human La Antigen: Conserved and Divergent Features of Structure and Function. Mol. Cell. Biol. 21, 367-379 (2001).

38. Fairley, J. A. et al. Human La is found at RNA polymerase III-transcribed genes in vivo. Proc Natl Acad Sci USA 102, 18350-18355 (2005).

39. Kurilla, M. G. \& Keene, J. D. The leader RNA of vesicular stomatitis virus is bound by a cellular protein reactive with anti-La lupus antibodies. Cell 34, 837-845 (1983).

40. Kurilla, M. G., Cabradilla, C. D., Holloway, B. P. \& Keene, J. D. Nucleotide sequence and host La protein interactions of rabies virus leader RNA. J Virol 50, 773-778 (1984).

41. Wilusz, J. \& Keene, J. D. Interactions of plus and minus strand leader RNAs of the New Jersey serotype of vesicular stomatitis virus with the cellular La protein. Virology 135, 65-73 (1984).

42. Wilusz, J., Kurilla, M. G. \& Keene, J. D. A host protein (La) binds to a unique species of minus-sense leader RNA during replication of vesicular stomatitis virus. Proc Natl Acad Sci USA 80, 5827-5831 (1983).

43. Bitko, V., Musiyenko, A., Bayfield, M. A., Maraia, R. J. \& Barik, S. Cellular La protein shields nonsegmented negative-strand RNA viral leader RNA from RIG-I and enhances virus growth by diverse mechanisms. J Virol 82, 7977-7987 (2008).

44. Domitrovich, A. M., Diebel, K. W., Ali, N., Sarker, S. \& Siddiqui, A. Role of La autoantigen and polypyrimidine tract-binding protein in HCV replication. Virology 335, 72-86 (2005).

45. Costa-Mattioli, M., Svitkin, Y. \& Sonenberg, N. La Autoantigen Is Necessary for Optimal Function of the Poliovirus and Hepatitis C Virus Internal Ribosome Entry Site In Vivo and In Vitro. Mol. Cell. Biol. 24, 6861-6870 (2004).

46. Liu, Y. et al. Autoantigen La Promotes Efficient RNAi, Antiviral Response, and Transposon Silencing by Facilitating MultipleTurnover RISC Catalysis. Mol Cell 44, 502-508 (2011).

47. Garcin, D., Itoh, M. \& Kolakofsky, D. A Point Mutation in the Sendai Virus Accessory C Proteins Attenuates Virulence for Mice, but Not Virus Growth in Cell Culture. Virology 238, 424-431 (1997).

48. Itoh, M., Shibuta, H. \& Homma, M. Single amino acid substitution of Sendai virus at the cleavage site of the fusion protein confers trypsin resistance. J Gen Virol 68 (Pt 11), 2939-2944 (1987). 
49. Yamaguchi, R., Iwai, H. \& Ueda, K. Variation of virulence and other properties among Sendai virus strains. Microbiol Immunol 32, 235-240 (1988).

50. Mochizuki, Y., Tashiro, M. \& Homma, M. Pneumopathogenicity in mice of a Sendai virus mutant, TSrev-58, is accompanied by in vitro activation with trypsin. J Virol 62, 3040-3042 (1988).

51. Lopez, C. B., Garcia-Sastre, A., Williams, B. R. \& Moran, T. M. Type I interferon induction pathway, but not released interferon, participates in the maturation of dendritic cells induced by negative-strand RNA viruses. J Infect Dis 187, 1126-1136 (2003).

52. Lopez, C. B. et al. TLR-independent induction of dendritic cell maturation and adaptive immunity by negative-strand RNA viruses. J Immunol 173, 6882-6889 (2004).

53. Baum, A. \& García-Sastre, A. Differential recognition of viral RNA by RIG-I. Virulence 2, 166-169 (2011).

54. Baum, A., Sachidanandam, R. \& García-Sastre, A. Preference of RIG-I for short viral RNA molecules in infected cells revealed by next-generation sequencing. Proceedings of the National Academy of Sciences of the United States of America 107, 16303-16308 (2010).

55. Melchjorsen, J. et al. Activation of innate defense against a paramyxovirus is mediated by RIG-I and TLR7 and TLR8 in a cell-typespecific manner. J Virol 79, 12944-12951 (2005).

56. Meerovitch, K. et al. La autoantigen enhances and corrects aberrant translation of poliovirus RNA in reticulocyte lysate. J Virol 67, 3798-3807 (1993).

57. Brierley, M. M., Marchington, K. L., Jurisica, I. \& Fish, E. N. Identification of GAS-dependent interferon-sensitive target genes whose transcription is STAT2-dependent but ISGF3-independent. FEBS J 273, 1569-1581 (2006).

58. Ank, N. et al. Lambda interferon (IFN-lambda), a type III IFN, is induced by viruses and IFNs and displays potent antiviral activity against select virus infections in vivo. J Virol 80, 4501-4509 (2006).

59. Coccia, E. M. et al. Viral infection and Toll-like receptor agonists induce a differential expression of type I and lambda interferons in human plasmacytoid and monocyte-derived dendritic cells. Eur J Immunol 34, 796-805 (2004).

60. Gack, M. U., Nistal-Villan, E., Inn, K.-S., Garcia-Sastre, A. \& Jung, J. U. Phosphorylation-Mediated Negative Regulation of RIG-I Antiviral Activity. J. Virol. 84, 3220-3229 (2010).

61. Gao, D. et al. REUL Is a Novel E3 Ubiquitin Ligase and Stimulator of Retinoic-Acid-Inducible Gene-I. PLoS ONE 4, e5760 (2009).

62. Oshiumi, H., Matsumoto, M., Hatakeyama, S. \& Seya, T. Riplet/RNF135, a RING finger protein, ubiquitinates RIG-I to promote interferon-beta induction during the early phase of viral infection. J Biol Chem 284, 807-817 (2009).

63. Sun, Z., Ren, H., Liu, Y., Teeling, J. L. \& Gu, J. Phosphorylation of RIG-I by Casein Kinase II Inhibits Its Antiviral Response. J. Virol. 85, 1036-1047 (2011).

64. Poeck, H. et al. Recognition of RNA virus by RIG-I results in activation of CARD9 and inflammasome signaling for interleukin 1 beta production. Nature immunology 11, 63-69 (2010).

65. Ling, Z., Tran, K. C. \& Teng, M. N. Human respiratory syncytial virus nonstructural protein NS2 antagonizes the activation of beta interferon transcription by interacting with RIG-I. J Virol 83, 3734-3742 (2009).

66. Goswami, R. et al. Viral degradasome hijacks mitochondria to suppress innate immunity. Cell Res 23, 1025-1042 (2013).

67. Pruijn, G. J., Thijssen, J. P., Smith, P. R., Williams, D. G. \& Van Venrooij, W. J. Anti-La monoclonal antibodies recognizing epitopes within the RNA-binding domain of the La protein show differential capacities to immunoprecipitate RNA-associated La protein. Eur J Biochem 232, 611-619 (1995).

68. Villenave, R. et al. Cytopathogenesis of Sendai Virus in Well-Differentiated Primary Pediatric Bronchial Epithelial Cells. Jsournal of Virology 84, 11718-11728 (2010).

69. Touzelet, O. et al. De novo generation of a non-segmented negative strand RNA virus with a bicistronic gene. Virus research 140, $40-48$ (2009).

70. Livak, K. J. \& Schmittgen, T. D. Analysis of relative gene expression data using real-time quantitative PCR and the 2(-Delta Delta C(T)) Method. Methods 25, 402-408 (2001).

71. Laemmli, U. K. Cleavage of structural proteins during the assembly of the head of bacteriophage T4. Nature 227, 680-685 (1970).

\section{Acknowledgements}

We would like to thank Dr. Kate Fitzgerald, Dr. Jacen Maier-Moore and Dr. Karl Albert Brokstad for plasmid constructs. This work was supported by the Irish Research Council, Science Foundation Ireland (Grant 08/IN.1/ B2091), the Public Health Agency Health and Social Care Research and Development Division, Northern Ireland, and the Northern Ireland Chest Heart and Stroke, who had no role in study design, data collection and analysis, decision to publish, or preparation of the manuscript.

\section{Author Contributions}

R.M. was responsible for study design, data collection, data analysis and drafting of the manuscript. L.B. was responsible for data collection, data analysis and manuscript revision. U.F.P. was involved in the coordination of the project, data analysis and critical revision of the manuscript. J.M.M. supplied reagents, helpful discussion and critical review of the manuscript. C.A.J. was responsible for initial conception and design of the study, data analysis and critical revision of the manuscript.

\section{Additional Information}

Supplementary information accompanies this paper at https://doi.org/10.1038/s41598-017-15197-9.

Competing Interests: The authors declare that they have no competing interests.

Publisher's note: Springer Nature remains neutral with regard to jurisdictional claims in published maps and institutional affiliations.

Open Access This article is licensed under a Creative Commons Attribution 4.0 International

License, which permits use, sharing, adaptation, distribution and reproduction in any medium or format, as long as you give appropriate credit to the original author(s) and the source, provide a link to the Creative Commons license, and indicate if changes were made. The images or other third party material in this article are included in the article's Creative Commons license, unless indicated otherwise in a credit line to the material. If material is not included in the article's Creative Commons license and your intended use is not permitted by statutory regulation or exceeds the permitted use, you will need to obtain permission directly from the copyright holder. To view a copy of this license, visit http://creativecommons.org/licenses/by/4.0/.

(C) The Author(s) 2017 